\title{
Estudio de las competencias de un director para un liderazgo efectivo en sectores vulnerables en la comuna de Antofagasta
}

Study of a headmaster's skills for an effective leadership in vulnerable areas of Antofagasta city

Studie über die Kompetenzen eines Direktors für effektive Führung in gefährdeten Sektoren in der Gemeinde Antofagasta

Étude des compétences d'un directeur pour un leadership efficace dans les secteurs vulnérables dans la commune d'Antofagasta

Estudo das competências de um diretor para uma liderança efetiva em setores vulneráveis na comuna de Antofagasta

Studio delle competenze di un direttore per una leadership efficace nei settori vulnerabili nel comune di Antofagasta Изучение компетенции директора для эффективного руководства в уязвимых секторах в общине Антофагаста

アントファガスタのコミューンにおける脆弱なセクターにおける効果的なリーダーシップのた めのディレクターの能力に関する研究 研究安托法加斯塔市镇弱势部门有效领导的主任的能力。

\author{
Gina Vera Erices \\ Universidad Internacional Iberoamericana (Chile) \\ judeagv@gmail.com
}

Fecha recepción: 07/10/2020

Páginas 9-35

Fecha aceptación: 29/12/2020

\section{Resumen.}

La investigación muestra que el liderazgo educativo es un factor de primer orden en la mejora de los resultados, evidenciando que es posible identificar ciertas características comunes de los directores que obtienen un buen desempeño y que son de vital importancia para influir en la mejora de las prácticas docentes. Necesitamos hoy, más que nunca, investigaciones que identifiquen el perfil y las prácticas de liderazgos efectivos de los directores exitosos para asegurar el cambio educativo, y que puedan contribuir a mejorar la calidad de educación de nuestros estudiantes. Esta investigación se planteó como objetivo determinar qué competencias debe tener un director para liderar una escuela efectiva en sectores vulnerables de la comuna de Antofagasta. La investigación se realizará a través del método de investigación cuantitativo. El objeto de estudio será el director y su liderazgo. La técnica de recolección de datos será un cuestionario aplicado en dos establecimientos municipales. Los datos obtenidos de las encuestas, serán codificados de acuerdo a las dimensiones del liderazgo a la que pertenecen y se analizarán los resultados de la investigación a través de métodos estadísticos, de los cuales se inferirán los resultados del estudio y el análisis descriptivo de los mismos.

Palabras claves: escuela efectiva; calidad; vulnerabilidad social; liderazgo educativo; gestión: dirección escolar 


\begin{abstract}
.
This research demonstrates that educational leadership is a key factor in the improvement of results, it is evidenced that it is possible to identify certain common traits in the headmaster that obtain good performances and that are vital to foster the improvement of teaching practices. Today, more than ever, we require researches that identify the profile and practices of effective leadership in successful headmasters, in order to ensure an educational change and that can contribute to improve the quality of the education in our students. This research set as objective to determine what competences a headmaster must have in order to manage an effective school in vulnerable sectors of the community of Antofagasta. The approach of this research will be performed through qualitative method with a descriptive method. The subject of study of this research is the headmaster and his/her leadership. The technique of this research will be data collection through surveys applied in two public schools. Data obtained from the surveys will be processed according to the domain of leadership they belong, and the results from the research will be analysed through statistical methods, from which the results of the study will be concluded and then proceed to perform descriptive analyses.
\end{abstract}

Keyword: Effective school; quality; social vulnerability; educational leadership; management; school direction

\title{
1.-Introducción.
}

El gran desafío de la escuela contemporánea es impartir una educación de calidad. Es por esta razón que se ha prestado gran importancia a la gestión educativa donde ha quedado de manifiesto que el liderazgo del director juega un rol crucial, ya que es la clave para generar un contexto adecuado para una educación de calidad, su rol debe ser de carácter pedagógico, es decir debe ejercerse en función del aprendizaje de los estudiantes con el fin de lograr los fines que la organización persigue.

En la revisión de las investigaciones llevadas a cabo en Chile dan cuenta de que el liderazgo ha sido un tema que no ha estado ausente en las investigaciones sobre educación y que han encontrado resultados tanto sobre la importancia del liderazgo como acerca de aquello que caracteriza a los directores efectivos y su influencia en los resultados escolares, ya sean medidos en términos académicos u organizacionales.

La literatura sobre el liderazgo escolar pone un marcado énfasis en los resultados académicos de los estudiantes (Hernández-Castilla, Murillo e Hidalgo, 2017; Murillo y Hernández-Castilla, 2015; Day y otros, 2011, 2009; Leithwood y Day, 2008; Leithwood, 2006). Tanto la política educativa chilena como los autores más citados en liderazgo ponen el foco en la importancia de los directivos en el desarrollo de los aprendizajes curriculares. Hay un énfasis excesivo en los directores como líderes individuales (Ainscow y West, 2008; Day, 2005; Gómez-Hurtado, 2012; Harris y Chapman, 2004; Mujis, Harris, Chapman, Stoll y Russ, 2004; León, 2001). Si bien los directores escolares son claves, como personas solitarias no siempre resultan relevantes para el cambio escolar. En los últimos años, los estudios sobre liderazgo escolar han estado enfocados principalmente en las prácticas en lugar de las competencias (MINEDUC, 2015). En este sentido, lo que más se rescata para 
la investigación de liderazgo, es la descripción de prácticas, es decir, de aquello que los directores de escuelas exitosas suelen hacer.

Los resultados de las distintas mediciones externas que se aplican actualmente a los establecimientos educacionales, ya sean nacionales (SIMCE) o internacionales (TIMS), reflejan que los logros de los aprendizajes esperados en el estudiantado chileno, en general, son deficitarios. Por lo cual los centros educativos deben impulsar un sistema de mejoramiento de la calidad de la educación que imparten, para lo cual además es necesario señalar que las escuelas que tienen buenos resultados escolares, a pesar de estar ubicadas en contexto vulnerables, tienen equipo directivos fuertes y comprometidos (Bellei, Morawietz, Valenzuela y Vanni, 2015; Agencia de Calidad de la Educación, 2017). La preocupación por el liderazgo educativo en Chile es un hecho relativamente reciente. Desde 2005 en adelante la política educativa ha dado un giro importante, pasando desde una concepción centralizada, en la que el ministerio entregaba lineamiento y condiciones básicas para la mejora, a una que reconoce la escuela como unidad de cambio y que descentraliza la responsabilidad por el mejoramiento escolar, dando mayor protagonismo y responsabilidad de apoyo a equipos directivos, liderazgo del director y a otros actores. En virtud de este cambio de enfoque, el MINEDUC queda enmarcado como una entidad que regula y asegura condiciones para que los establecimientos puedan llevar a cabo sus iniciativas de mejora.

De acuerdo a Leithwood et al. (2008), el liderazgo directivo sería la segunda variable interna de la escuela que influye más en los resultados de los estudiantes, después del trabajo de aula de los docentes. Al interior de las escuelas, el liderazgo directivo puede contribuir a la mejora escolar mediante su influencia sobre las condiciones y las capacidades de los docentes, propiciando las iniciativas de cambio de manera coherente y sostenible. Nace la interrogante sobre, qué competencias debe tener un director para promover una escuela efectiva en sectores más vulnerables y si el liderazgo transformacional con sus prácticas en el establecimiento permite incidir positivamente en el trabajo de los profesores y en los aprendizajes de los alumnos de escuelas municipalizadas. El objetivo de esta tesis es determinar qué competencias de gestión y liderazgo debe tener un director para liderar una escuela efectiva en sectores vulnerables de la comuna de Antofagasta. Para ello trabajamos con tres modelos aportados, de Keneth Leithwood, Fundación Chile y Ministerio de Educación (SACGE) Sistema de Aseguramiento de Calidad y Gestión Escolar, que postula que el liderazgo directivo influye en el rendimiento de los estudiantes de manera indirecta, esto es, mediado por el trabajo de los docentes. En otras palabras, el liderazgo, a través de ciertas prácticas o comportamientos de los directivos, influye en el trabajo de los docentes, y desde ahí, en el de los estudiantes. Para lograr los propósitos antes enunciados, las escuelas efectivas deben partir por realizar un diagnóstico muy completo y en todas sus áreas o estamentos organizacionales, para que de acuerdo a los resultados que arroje ese diagnóstico se elaboren estrategias para instalar los cambios que la escuela requiera.

Para la investigadora, este estudio se caracteriza por su conveniencia e implicaciones prácticas; criterios que según Hernández Sampieri y otros (2000), deben existir para establecer las bases sólidas que permiten justificar la realización de una investigación. Una de las principales aportaciones es que la metodología que se utilice, servirá de base para otras investigaciones, ya sea en otras escuelas municipales de Antofagasta o para los 
establecimientos de otras comunas de la II región y las autoridades de la Corporación Municipal de Desarrollo Social de Antofagasta, como los directores de los establecimientos analizados, podrán focalizar los planes de acción para el fortalecimiento o desarrollo de las competencias requeridas por los directivos.

\section{2.-Importancia del liderazgo de los directores escolares.}

El liderazgo escolar se ha posicionado como uno de los temas emergentes de las últimas décadas en materia de educación y cambio escolar. Según Leithwood, Harris y Hopkins (2008), el liderazgo escolar es el segundo factor que más influye en el aprendizaje de los estudiantes (después del trabajo en aula) y que no depende precisamente del carisma, sino de las prácticas, entendiendo el liderazgo como "la labor de movilizar e influenciar a otros para articular y lograr los objetivos y metas compartidas" (Leithwood et al., 2006). No obstante, el liderazgo es una función más amplia que la labor ejercida por el director del establecimiento y es compartida con otras personas de la institución (MINEDUC, 2015). Sin necesidad de hacer un profuso recorrido histórico, conviene dejar evidencias de la relevancia que ha adquirido en los últimos 30 años en Chile (Weinstein y Muñoz, 2012). Durante mucho tiempo se ha venido estudiando la historia de las reformas educativas y es un hecho que cada una está plagada de fracasos donde se observa que quienes han estado encargados de la elaboración de políticas, generalmente no valoran bien hasta qué punto los docentes y líderes escolares cuentan con los conocimientos y habilidades necesarias para llevar a cabo lo que se les pide. Una real mejora en las escuelas exige cambios fundamentales en el diseño y liderazgo de éstas y de los sistemas escolares. Supone un cambio en los valores y normas acerca de cómo los profesores y directores conciben los objetivos de su trabajo, y cambios en quienes pensamos son los líderes de las escuelas, dónde están y lo que hacen, y cambios en los requerimientos de conocimientos y destrezas para un buen trabajo escolar, es necesario hacer transformaciones profundas en las escuelas para que estas sean un verdadero lugar de aprendizaje. Es importante que tanto alumnos, profesores y directivos se responsabilicen y sean capaces de dar cuenta de forma permanente de los resultados que se obtienen, con el propósito de mejorar nuestro sistema educativo, Elmore (2006, p. 10) señala "no hay nada más práctico que una buena teoría". El propósito es sugerir que las reformas escolares, cuyo objetivo es mejorar los aprendizajes de los alumnos, requieren una teoría sobre las prácticas, que se funde en la labor que realizan las personas en las escuelas, debiendo preceder y alimentar las políticas. En este sentido, el éxito de las reformas educativas depende de la habilidad de los directores y profesores para construir potentes "teorías de la acción", que les permitan entender de qué manera sus acciones afectan a lo que aprenden los alumnos.

Según Elmore (2006, p. 13) "el liderazgo consiste esencialmente en crear, alimentar y desarrollar la capacidad de los docentes y estudiantes para que se involucren en un aprendizaje de alto nivel y para que reflexionen sobre su propia práctica de aprendizaje". La labor de cualquier docente, ya sea el director de una escuela liderando un grupo de profesores, un instructor trabajando con un grupo de docentes o un profesor trabajando con un grupo de estudiantes, es transferir el control de aprendizaje del profesor al estudiante. De igual modo, liderar es crear las condiciones para que otros asuman el control de su propio aprendizaje, tanto a nivel individual como colectivo. También es importante enseñar, de manera más o menos directa, los conocimientos y destrezas necesarios para ser un buen aprendiz. Pero en última instancia, la eficacia tanto del líder como del profesor no está 
determinada por cuán bien transfieran su propio conocimiento a otras personas, sino de qué forma prepara a otros para que asuman el control de su propio aprendizaje.

El liderazgo en términos generales ha sido conceptualizado de muchas formas. Frecuentemente se encuentra presente en la investigación internacional la idea de que el liderazgo tiene que ver con la "influencia". Así, el liderazgo consistiría en la capacidad de plantear principios de modo que sean tomados por otros como propios, creándose así un sentido compartido que moviliza a la organización en pro de objetivos o metas comunes. Siguiendo a Leithwood, Day, Sammons y Hopkins (2006), el liderazgo se caracteriza por fijar un "norte" para la organización, y tener la capacidad de movilizarla en esa dirección. Si a este liderazgo le agregamos la palabra educativo, entonces podemos complementar la definición y, tomando las palabras de Robinson (2009), decir que el liderazgo educativo es aquel que influye en otros para hacer las cosas que se espera que mejoren los resultados de aprendizaje de los estudiantes. Los líderes responsables saben que el éxito está en hacer buen uso estratégico de recursos para mejorar el desempeño, lo que a su vez les va a permitir desarrollar capacidades. Los líderes deben ser estratégicos para posicionar la organización hacia dónde quieren llegar.

La investigación y la experiencia han evidenciado que el comportamiento y la actitud de la persona que asume las funciones de dirección en la escuela son un elemento fundamental que determina la existencia, la calidad y el éxito de los procesos de cambio en la escuela (Murillo, 2008). De esta forma, es posible afirmar que, si queremos cambiar las escuelas y, con ello, mejorar la educación, necesitamos personas que tengan un real compromiso y que puedan ejercer un liderazgo desde el interior de la escuela que impulse, gestione, coordine y dinamice el proceso de transformación. El liderazgo escolar cobra importancia fundamental en la cultura escolar en y desde el equipo directivo y no solo desde los directores (Bellei, Muñoz y Raczynski, 2004; Eyzaguirre y Fontaine, 2008). Esto implica, además, dejar de apostar tanto en el virtuosismo de los directores y empezar a pensar el liderazgo como un tema de equipos directivos. Algunos estudios sostienen que los cargos directivos, independiente de la forma de ejercer el liderazgo, se llevan a cabo de forma rutinaria y con un marcado énfasis administrativos, sin direccionar decisiones hacia la transformación escolar y/o cultural (Carbone, Olguín, Ugalde y Sepúlveda, 2008; López, Ahumada, Galdámez y Madrid, 2012).

En Chile, los estudios de Bellei, Valenzuela, Vanni y Contreras (2014) sobre trayectorias de mejoramiento escolar, encontraron que se producen procesos de "reculturización" en aquellas escuelas que mejoran, siendo la comunidad y valores algunos de los niveles de este cambio cultural; elementos cruciales en una cultura inclusiva (Booth y Ainscow, 2015). Conviene mencionar que este proceso es liderado por los equipos directivos. Por esto mismo, se hace necesario y urgente consolidar en las escuelas equipos directivos que bajo un contexto de alta demanda laboral, ejerzan un liderazgo educativo que contribuya a la transformación de las comunidades escolares y no solamente con foco en rendimiento escolar (Gómez-Hurtado, 2012; Bolivar, 2010; Marfán, Muñoz y Weinstein, 2012). A la vez, la consolidación de un buen equipo directivo es pieza clave en el cambio cultural (Ahumada et al., 2009). Hoy existe abundante investigación que ha demostrado una correlación clara entre el liderazgo del director y los resultados de aprendizaje de los alumnos de un establecimiento educacional. Del trabajo de los autores Hendricks (2000) y National Association of Secondary School Principáis (2002), se desprende que este liderazgo supone 
aptitudes tales como: capacidad de crear ambientes donde profesores y estudiantes se sientan seguros y donde exista orden y estructura, capacidad de fijar una visión y metas claras qué se focalicen en altos niveles de rendimiento escolar, capacidad de tomar decisiones pensando en cuáles serán sus efectos en los resultados de aprendizaje de los alumnos, altas expectativa en lo que pueden hacer los profesores y los estudiantes y una constante motivación hacia ellos para mejorar, y capacidad de involucrarse en los temas del currículo y los métodos de enseñanza aprendizaje.

La literatura sobre liderazgo escolar pone un marcado énfasis en los resultados académicos de los estudiantes (Hernández-Castilla, Murillo e Hidalgo, 2017; Murillo y HernándezCastilla, 2015; Day y otros, 2011; Day y otros, 2009; Leithwood y Day, 2008; Leithwood, 2006). Tanto la política educativa chilena como los autores más citados en liderazgo ponen el foco en la importancia de los directivos en el desarrollo de los aprendizajes curriculares. Hay un énfasis excesivo en los directores como líderes individuales (Ainscow y West, 2008; Day, 2005; Gómez-Hurtado, 2012; Harris y Chapman, 2004; Muijs, Harris, Chapman, Stoll \&Russ, 2004; León, 2001). Si bien los directores escolares son claves, como personas solitarias no siempre resultan relevantes para el cambio escolar, por lo tanto, conviene avanzar en la búsqueda de nuevos líderes y en la relevancia de los equipos directivos. En los últimos años, los estudios sobre liderazgo escolar han estado enfocados principalmente en las prácticas en lugar de las competencias (MINEDUC, 2015). Algunos autores (Uribe y Celis, 2012; Carroll, Levy y Richmond, 2008; Chia \& Holt, 2006; Spillane, 2005) mencionan diferencias importantes entre "práctica" y "competencia" en el ámbito del liderazgo. Principalmente sostienen que las prácticas son compartidas, contextualizadas, flexibles y relacionales. A diferencia de las competencias, que son individuales y que suponen que funcionan en todo contexto sin considerar la acción situada.

Antes de describir qué se entiende por competencia de un director de establecimiento educacional, se analizarán las definiciones de determinados investigadores del tema. Frade (2008, p. 26) señala "capacidad adaptativa, cognitiva y conductual para responder adecuadamente a las demandas que se presentan en el entorno. Es un saber pensar para poder hacer frente a lo que se necesita" $\operatorname{OCDE}(2002$, p. 9). Capacidad de responder a demandas complejas y llevar a cabo tareas diversas de forma adecuada. Supone una combinación de habilidades prácticas, conocimiento, motivación, valores éticos, actitudes, emociones y otros componentes sociales y de comportamientos que se movilizan conjuntamente para lograr una acción eficaz.

El liderazgo escolar, después del trabajo en el aula, es el segundo factor intraescuela que más influye en el aprendizaje de los estudiantes (Leithwood et al., 2006) y que no depende precisamente del carisma, sino de las prácticas, entendiendo el liderazgo como la labor de movilizar e influenciar a otros para articular y lograr los objetivos y metas compartidas (Leithwood, Harris y Hopkins, 2008, Leithwood et al., 2006). No obstante, el liderazgo es una función más amplia que la labor ejercida por el director del establecimiento y es compartida con otras personas de la institución (Harris, Ng, Jones, Than, 2018; Vaillant, 2018; Mineduc, 2015). De esta forma, el liderazgo escolar se configura como un conjunto de prácticas que presenta variaciones de acuerdo al contexto (Pam et al., 2011; Marfán, Muñoz y Weinstein, 2012; Ahumada, Galdames y Clarke, 2015). El concepto de prácticas de liderazgo comprende un constructo dinámico y contextualizado (Uribe y Celis, 2012). Según Spillane y Ortiz (2017) las prácticas de liderazgo surgen a partir de las interacciones 
entre los miembros de una escuela en función de los intereses comunes y considerando el contexto. Es decir, los individuos actúan pero lo hacen en relación a otros. Así, la práctica se constituye a partir de una colaboración entre las personas que trabajan en una organización, por ello su definición también está influida por el entorno, lo que la distingue de la simple descripción de un saber o un hacer descontextualizado (Spillane, 2005). Desde esta perspectiva, el liderazgo es la práctica del mejoramiento (Elmore, 2010) y no se trata de atributos personales, sino de un conjunto de acciones, que tienen su fundamento en conocimientos, habilidades y hábitos que pueden ser enseñados y aprendidos (equipo directivo).

No obstante, los estudios sobre prácticas de liderazgo siguen siendo pocos, debido en parte a la importancia que se le ha dado a las competencias (Mineduc, 2015) y debido al cambio en las políticas públicas en la última década en materia de liderazgo escolar (Day et al., 2009; Marfán et al. 2012, Ahumada et al., 2015). Según Leithwood, Louis, Anderson y Wahlstrom (2004), el desafío en liderazgo escolar es comprometerse en las prácticas, pues de esta manera nos acercamos a las escuelas considerando sus características propias. Los enfoques socioculturales nos permiten entender las prácticas como acciones situadas culturalmente construidas (Martínez, 1999), reconociendo que estas se encuentran distribuidas y que no habitan en una sola persona. Desde los planteamientos de las comunidades de prácticas (Lave y Wenger, 1991; Wenger, 2001) aprender significa participar de una comunidad y, por lo tanto, las prácticas (y el aprendizaje) son posibles cuando un grupo de personas comparten un interés, un conjunto de problemas o una pasión sobre un tema. Asumir un enfoque sociocultural basado en la teoría de las comunidades de práctica implica involucrarse en los valores de las instituciones y en las culturas que la sustentan (Moliner, Sales y Escobedo, 2016).

\section{3.-Metodología.}

El objeto de estudio para esta investigación, fue el director y sus prácticas de liderazgo. Por confidencialidad, los establecimientos se denominaron como "A" y "B". La investigación, se basó en el paradigma positivista, de carácter descriptivo y con una metodología cuantitativa, donde se emplearon procesos cuidadosos sistemáticos y empíricos, lo cual nos permite generar un nuevo conocimiento o demostrar una nueva teoría. Se especificará si se acepta o rechaza la hipótesis. La técnica de recolección de datos fue a través de dos cuestionarios de elaboración propia y registro de datos estadísticos de pruebas estandarizadas, como el SIMCE (Sistema de Medición de la calidad de la Educación). El cual nos permitió reunir información sobre los hechos. Los cuestionarios aplicados fueron validados a través de expertos y también a través de análisis estadísticos, para dar fiabilidad y validez. La muestra utilizada fue aleatoria, simple, dirigida y está representada con el $20 \%$ del universo, correspondiente a 322 encuestados. Los diferentes actores que participaron fueron: equipo directivo, docentes, estudiantes, padres y apoderados. El muestreo fue de carácter intencional, dinámico y secuencial. Está conformada por dos directores de establecimientos educacionales municipalizados de la comuna de Antofagasta, que imparten enseñanza básica y han rendido prueba SIMCE.

El análisis de datos se realizó a través de un programa computacional SSPS, aplicado por experto en el área, donde se analizaron los datos mediante preparación de la base de datos, comprobación de las propiedades psicométricas de los cuestionarios, realización de análisis 
descriptivos de los resultados, comprobación de la homogeneidad de los establecimientos, realización de inferencias sobre los resultados y sistematización de los resultados (de los procedimientos aplicados), mediante la consolidación de tablas, gráficos, figuras, diagramas o utilizando cualquier tipo de representación que favorezca una fácil interpretación de lo que se ha conseguido a lo largo del estudio, se pretende que sea claro para el lector y para quien desea conocer qué se hizo con los datos y cómo fueron analizados, además de la presentación de un informe. Por todo lo anteriormente expuesto se considera, el método de investigación cuantitativa el más adecuado a los propósitos de esta investigación.

\section{4.-Resultados.}

4.1.- Primer informe del proceso de análisis y entrega de resultados de los análisis estadísticos realizados sobre las respuestas a los cuestionarios aplicados.

El presente informe da cuenta del proceso de análisis y entrega resultados de los análisis estadísticos realizados sobre las respuestas a los cuestionarios aplicados a integrantes de la comunidad escolar de dos establecimientos de la ciudad de Antofagasta. Dichos cuestionarios pretenden medir las competencias de liderazgo de los directores de estas escuelas y la aplicación de esta encuesta se encuentra en el marco de una investigación de tesis doctoral, cuyo objetivo es determinar qué competencias debe tener un director para liderar una escuela efectiva en sectores vulnerables de la comuna de Antofagasta.

En este estudio, se analizaron los modelos de liderazgos tales como el modelo de la Fundación Chile, Modelo de liderazgo del Ministerio de Educación (SACGE) y el modelo Transformacional de Leithwood, donde se concluye que la escuela efectiva requiere de un adecuado liderazgo del director, el que se concreta a través de determinadas prácticas, donde al comparar los tres modelos se resumieron en seis dimensiones. Por lo anterior, se analizarán las dimensiones y las prácticas de liderazgos a través de un tipo de investigación descriptiva, en las Escuelas Municipales A y B, de la comuna de Antofagasta. Las dimensiones del primer cuestionario son las siguientes: 1) Si el director está siempre centrado en las necesidades de los alumnos; 2) Si la dirección es capaz de transmitir los fines y prioridades educativas a toda la comunidad escolar; 3) Si el director apoya a los profesores en el ámbito curricular; 4) Si la dirección crea un ambiente ordenado y un clima escolar que facilita la enseñanza y el aprendizaje; 5) Si el director conoce la enseñanza de calidad y trabaja activamente con el profesorado para mejorar su capacidad profesional, 6) Si la dirección supervisa y evalúa el rendimiento de la escuela.

Con respecto al segundo cuestionario, se midieron las siguientes dimensiones: 1)Liderazgo carismático, 2)Consideración individualizada, 3)Liderazgo inspiracional, 4)Estimulación intelectual. Este informe está abocado en presentar lo realizado en la primera fase del trabajo, enfocado en la preparación de las bases de datos y el análisis de las propiedades psicométricas del cuestionario. Para el desarrollo de estos temas, el texto se encuentra dividido en secciones y subsecciones, siendo el análisis de las propiedades psicométricas la sección más extensa pues da cuenta de diversos análisis estadísticos aplicados a los dos instrumentos que fueron aplicados.

En primer lugar, se midió la fiabilidad de ambos cuestionarios, de manera global y en cada dimensión. Para tal efecto, se calculará el estadístico Kuder - Richardson 20 (KR20), un 
índice de consistencia interna equivalente al alfa de Cronbach para escalas con respuestas dicotómicas (DeVellis, 2017). Se establecerán niveles de aceptabilidad aquellos coeficientes mayores a 0,8 para la escala global y mayores a 0,6 para las dimensiones. De ser necesario, se eliminarán algunos ítems que permitan mejorar la fiabilidad del instrumento de medición. En segundo lugar, se realizarán análisis descriptivos sobre las respuestas a los cuestionarios. Para la ejecución de estos procedimientos, será calculado, para cada encuestado, un índice de la escala global e índices de cada dimensión del cuestionario. Estos índices consisten en la proporción de respuestas afirmativas entregadas a las diferentes aseveraciones que incluyen los cuestionarios aplicados. De tal manera, los índices o puntajes de cada persona serán números entre 0 y 1 , en donde 0 indica la ausencia total de respuestas afirmativas en los ítems de la escala y 1 la presencia de un $100 \%$ de respuestas positivas a dichos reactivos. Sobre estos índices serán realizados procedimientos estadísticos descriptivos, tales como generación de gráficos de frecuencia, cálculo de medidas de tendencia central y cálculo de medidas de dispersión (Quader, 2016).

Estos procedimientos se realizarán considerando toda la muestra según los dos tipos de instrumentos aplicados: el cuestionario para directivos y docentes y el cuestionario para alumnos y apoderados. Además, se realizarán los mismos análisis para cada escuela en particular, de forma general y en cada subgrupo de estudio; luego, se compararán los datos obtenidos entre establecimientos. Por último, se aplicarán procedimientos de inferencia estadística para determinar si existen diferencias significativas entre los índices de cada establecimiento y entre los subgrupos de estudio. Para ello, se efectuarán procedimientos para determinar si se cumplen los supuestos necesarios para realizar inferencia paramétrica. En aquellos casos en que puede advertirse que los requisitos están garantizados, se aplicarán pruebas $t$ de Student. Cuando los supuestos no puedan comprobase, se efectuarán procedimientos no paramétricos, en específico, la prueba $U$ de Mann - Whitney.

Para cada prueba, se fijó un nivel de significancia de 0,05 . Los valores $p$ asociados a cada contraste serán aproximados por redondeo a la tercera cifra decimal. Los índices, la generación de tablas de frecuencia, el cálculo de los estadísticos de tendencia central y dispersión y los procedimientos de inferencia serán calculados utilizando el programa IBM SPSS Statistics versión 21. Los gráficos de frecuencia serán realizados utilizando el programa LibreOffice Calc versión 6.

La escuela $A$ es un establecimiento que, en el nivel de enseñanza básica, posee 60 profesores y 1192 estudiantes, agrupados en 29 cursos de primero a octavo año. La escuela $B$, por otra parte, es un establecimiento que, en el nivel de enseñanza básica, posee 56 profesores y 1067 estudiantes, agrupados en 24 cursos de primero a octavo año (Ministerio de Educación, 2019a; Ministerio de Educación, 2019b).

En la última medición SIMCE comparable del año 2018 para el nivel de $4^{\circ}$ básico, la escuela A fue categorizada en el grupo socioeconómico "medio", la puntuación de los índices de desarrollo social y personal se encuentran a menos de una desviación estándar del promedio de los establecimientos municipales de la comuna de Antofagasta en las 4 dimensiones medidas (Autoestima académica y motivación, Clima de convivencia escolar, Participación y formación ciudadana y Hábitos de vida saludable). 
El establecimiento B, en cambio, fue categorizado en el grupo socioeconómico "medio bajo" y la puntuación de los índices de desarrollo personal y social también se encuentran a menos de una desviación estándar del promedio de establecimientos municipales de la comuna (Agencia de calidad de la educación, 2018).

La tabla 1 resume la información anterior y aporta algunas tasas útiles para comparar la similitud de ambas escuelas.

Tabla 1

Índices de caracterización de ambas escuelas y comparación con el promedio comunal

\begin{tabular}{llll}
\hline & Escuela A & Escuela B & Promedio \\
\hline Número de profesores & 60 & 56 & \\
Número de alumnos & 1192 & 1067 & \\
Número de cursos & 29 & 24 & \\
Tasa de alumnos por profesor & 19,9 & 19,0 & 20,1 \\
Tasa de alumnos por curso & 41,1 & 44,45 & $33,2(5,4)$ \\
GSE & Medio & Medio bajo & \\
AAM & 73,6 & 77,0 & $74,2(3,6)$ \\
CCE & 78,0 & 74,5 & $73,9(4,5)$ \\
PFC & 79,7 & 80,7 & $77,8(4,7)$ \\
HVS & 67,6 & 69,6 & $67,3(4,6)$ \\
IVE & 84,38 & 85,66 & 76,6 \\
\hline
\end{tabular}

Fuente propia

Nota: En el promedio fueron consideradas solo las escuelas básicas municipales de la comuna de Antofagasta. La desviación estándar aparece entre paréntesis si está disponible. GSE: Grupo socioeconómico asignado para la prueba SIMCE 2018 de $4^{\circ}$ básico, AAM: índice de autoestima académica y motivación, CCE: índice de clima de convivencia escolar, PFC: índice de participación y formación ciudadana, HVS: índice de hábitos de vida saludable, IVE: índice de vulnerabilidad escolar. El promedio IVE incluye educación media. Fuente: elaboración propia en base a datos Ministerio de Educación (2019), Agencia de calidad de la educación (2018) y JUNAEB (2019).

Comparando los índices de cada establecimiento, es posible afirmar que ambas escuelas son muy similares. El único indicador distinto es el de grupo socioeconómico, lo que podría advertir que la escuela A posee alumnos más aventajados que los de la escuela B. Sin embargo, el índice de vulnerabilidad escolar que mide el porcentaje de estudiantes vulnerables muestra que ambos establecimientos poseen similar porcentaje de alumnos en esta condición (Junta Nacional de Auxilio Escolar y Becas, 2019), sugiriendo que la composición socioeconómica de los estudiantes es bastante comparable.

\section{2.-Resultados descriptivos de las respuestas a las escalas aplicadas.}

En esta sección se entregarán los principales resultados de los análisis descriptivos realizados a las respuestas entregadas por directivos, profesores, estudiantes y apoderados de dos establecimientos educacionales de la comuna de Antofagasta.

La tabla 2 compara el promedio y la desviación estándar de los puntajes de la escala global del cuestionario para directivos y docentes entre ambos establecimientos. 
Tabla 2

Media y desviación estándar de los puntajes de la escala global del cuestionario para directivos y docentes en ambos establecimientos

\begin{tabular}{lll}
\hline Estadístico & Escuela A & Escuela B \\
\hline Media & 0,92 & 0,78 \\
Desviación estándar & 0,10 & 0,20 \\
\hline
\end{tabular}

Fuente propia

Se puede observar que el establecimiento $A$ presenta un promedio más alto que el establecimiento B. También es posible visualizar que el establecimiento B posee puntajes más heterogéneos, es decir, hay una visión más positiva de la labor directiva en el establecimiento A y esta visión positiva es homogénea dentro de los encuestados.

Los directivos obtienen índices más altos respecto de los profesores, tanto en el establecimiento A, como en el B. Sin embargo, en el establecimiento B existen brechas más amplias entre la opinión de directivos y docentes (ver tabla 3).

Tabla 3

Media y desviación estándar de los puntajes de la escala global en directivos y docentes de ambos establecimientos

\begin{tabular}{lllll}
\hline & Escuela A & & Escuela B & \\
& Directivos & Docentes & Directivos & Docentes \\
\hline Media & 0,97 & 0,90 & 0,94 & 0,73 \\
Desviación estándar & 0,04 & 0,11 & 0,04 & 0,21 \\
\hline
\end{tabular}

Fuente propia

Con respecto al cuestionario para alumnos y apoderados, es posible observar que los alumnos de la escuela $A$ tienen una mejor percepción del liderazgo del cuerpo directivo que los del establecimiento $B$, puesto que, en promedio, los puntajes obtenidos en la escala son más altos (ver tabla 4). No se visualizan grandes diferencias en la dispersión de los índices, puesto que la desviación estándar es bastante similar.

Tabla 4

Media y desviación estándar de los puntajes de la escala global del cuestionario para alumnos y apoderados en ambos establecimientos

\begin{tabular}{lll}
\hline & Escuela A & Escuela B \\
\hline Media & 0,89 & 0,83 \\
Desviación estándar & 0,14 & 0,13
\end{tabular}

Fuente propia

Si se analizan los subgrupos de la muestra, se pude visualizar que en la escuela A los alumnos obtuvieron, en promedio, puntajes más altos que los apoderados; por el contrario, en el establecimiento B son los apoderados quienes obtuvieron puntajes más altos que los 
estudiantes (ver tabla 5). En ambos establecimientos, la opinión de los alumnos es más homogénea que la de los apoderados.

Tabla 5

Media y desviación estándar de los puntajes de la escala global en directivos y docentes de ambos establecimientos

\begin{tabular}{lllll}
\hline & Escuela A & & Escuela B & \\
& Alumnos & Apoderados & Alumnos & Apoderados \\
\hline Media & 0,91 & 0,86 & 0,80 & 0,87 \\
Desviación estándar & 0,09 & 0,16 & 0,10 & 0,14 \\
\hline
\end{tabular}

Fuente propia

Otro factor interesante de destacar sobre el análisis anterior es la similitud en las medias de los apoderados de ambos establecimientos. Esto quiere decir que los apoderados de las dos escuelas valoran de la misma manera el liderazgo del cuerpo directivo. Por otra parte, los estudiantes sí presentan una diferencia importante en los promedios de los puntajes obtenidos, lo que permite concluir que la escuela $A$ posee un promedio más alto que el $B$ debido a la opinión de los alumnos de ese establecimiento.

4.3.-Segundo informe de análisis de resultados en investigación de tesis doctoral análisis descriptivo de los resultados de las respuestas a las escalas de medición aplicadas.

El presente informe entrega los resultados de los análisis descriptivos realizados a las respuestas entregadas por directivos, profesores, estudiantes y apoderados de dos establecimientos educacionales de la comuna de Antofagasta. Para la ejecución de los procedimientos, fue calculado, para cada encuestado, un índice de la escala global y de cada dimensión del cuestionario. Este índice consiste en la proporción de respuestas afirmativas entregadas a las diferentes aseveraciones que incluían los cuestionarios aplicados. Es decir, a cada persona se le calculó el índice de respuestas afirmativas, tanto para el cuestionario global, como para cada dimensión. Este índice se calcula: números de respuestas "si" / número de aseveraciones. Para cada cuestionario y cada dimensión se definieron categorías distintas dependiendo del número de aseveraciones y la distribución de los encuestados. Por eso hay cambios en los intervalos del eje $\mathrm{X}$ en diferentes gráficos. De tal manera, los índices o puntajes de cada persona serán números entre 0 y 1 , en donde 0 indica la ausencia total de respuestas afirmativas en los ítems de la escala y 1 la presencia de un $100 \%$ de respuestas positivas a dichos reactivos. Sobre estos índices fueron realizados procedimientos estadísticos descriptivos, tales como generación de gráficos de frecuencia, cálculo de medidas de tendencia central y cálculo de medidas de dispersión (Quader, 2016).

La presentación de resultados se encuentra dividida según los dos tipos de instrumentos aplicados: el cuestionario para directivos y docentes y el cuestionario para alumnos y apoderados. En cada apartado se mostrarán los principales resultados de cada escuela, de forma general y en cada subgrupo de estudio; luego, se compararán los datos obtenidos entre establecimientos. 
Los índices, la generación de tablas de frecuencia y los estadísticos de tendencia central y dispersión fueron calculados utilizando el programa IBM SPSS Statistics versión 21. Los gráficos de frecuencia fueron realizados utilizando el programa LibreOffice Calc versión 6. El presente informe fue redactado en el programa Word de Microsoft Office 365 ProPlus, versión 1902.

\section{4.-Comparaciones entre establecimientos $\mathrm{A}$ y $\mathrm{B}$ y dimensiones.}

La tabla 6 compara el promedio y la desviación estándar de los puntajes de la escala global entre ambos establecimientos

\begin{tabular}{lll}
\hline Estadístico & Escuela A & Escuela B \\
\hline Media & 0,92 & 0,78 \\
Desviación estándar & 0,10 & 0,20
\end{tabular}

Fuente propia

Se puede observar que el establecimiento A presenta un promedio más alto que el establecimiento B. También es posible visualizar que el establecimiento B posee puntajes más heterogéneos, es decir, hay una visión más positiva de la labor directiva en el establecimiento A y esta visión positiva es homogénea dentro de los encuestados. Al comparar entre dimensiones (ver tabla 7), es posible observar que en todas ellas los encuestados de la escuela A puntuaron, en promedio, más que aquellos de la escuela $B$. Se puede visualizar que en ambos establecimientos la dimensión 1 es aquella que tiene un promedio más alto. En el resto de las dimensiones, los promedios del establecimiento A son muy similares, mientras que en el establecimiento $B$ hay una tendencia a puntuar más alto en las dimensiones 2 y 4 por sobre las dimensiones 3,5 y 6 .

\section{Tabla 7}

Media de los puntajes de cada dimensión del cuestionario para directivos y docentes en ambos establecimientos

\begin{tabular}{lll}
\hline Dimensión & Escuela A & Escuela B \\
\hline Dimensión 1 & 0,99 & 0,90 \\
Dimensión 2 & 0,89 & 0,84 \\
Dimensión 3 & 0,92 & 0,77 \\
Dimensión 4 & 0,90 & 0,80 \\
Dimensión 5 & 0,91 & 0,76 \\
Dimensión 6 & 0,92 & 0,74 \\
\hline
\end{tabular}

Fuente propia

Las tablas 8 y 9 comparan los mismos estadísticos recién analizados, en cada uno de los subgrupos de la muestra.

Se puede comprobar lo que se había advertido al analizar los gráficos de distribuciones: Los directivos obtienen índices más altos en la escala global y en cada dimensión respecto de los profesores, tanto en el establecimiento A, como en el B. Sin embargo, en el 
establecimiento $B$ existen brechas más amplias entre la opinión de directivos y docentes (ver tabla 8).

Tabla 8

Media y desviación estándar de los puntajes de la escala global en directivos y docentes de ambos establecimientos

\begin{tabular}{lllll}
\hline & Escuela A & & Escuela B & \\
& Directivos & Docentes & Directivos & Docentes \\
\hline Media & 0,97 & 0,90 & 0,94 & 0,73 \\
Desviación estándar & 0,04 & 0,11 & 0,04 & 0,21 \\
\hline
\end{tabular}

Fuente propia

Analizando las dimensiones, puede observarse una disonancia entre la opinión de directivos y docentes del establecimiento A: La dimensión 6 presenta un promedio mucho más bajo que el resto de las dimensiones en las respuestas de directivos, mientras que en profesores es la segunda dimensión mejor puntuada (ver tabla 9).

\section{Tabla 9}

Media de los puntajes de cada dimensión de la escala en directivos y docentes de ambos establecimientos

\begin{tabular}{lllll}
\hline & Escuela A & & Escuela B & \\
& Directivos & Docentes & Directivos & Docentes \\
\hline Dimensión 1 & 1,00 & 0,98 & 1,00 & 0,86 \\
Dimensión 2 & 1,00 & 0,85 & 0,95 & 0,80 \\
Dimensión 3 & 0,98 & 0,90 & 0,91 & 0,72 \\
Dimensión 4 & 0,99 & 0,87 & 0,94 & 0,76 \\
Dimensión 5 & 0,98 & 0,88 & 0,95 & 0,69 \\
Dimensión 6 & 0,91 & 0,92 & 0,93 & 0,67 \\
\hline
\end{tabular}

Fuente propia

Además, existe una brecha importante en la dimensión 2 en el mismo establecimiento. En este caso, el $100 \%$ de los directivos respondió positivamente las aseveraciones, mientras que el promedio en profesores es el más bajo en comparación con el resto de las dimensiones.

En la sección anterior también se habían advertido brechas de opinión entre directivos y docentes del establecimiento $B$, específicamente en las dimensiones 5 y 6 , que se confirman al analizar las medias mostradas en la tabla 9. Al comparar entre establecimientos, se puede advertir que la dimensión peor evaluada por los directivos del establecimiento A es la 6 , mientras que los directivos de la escuela $B$ evalúan peor la dimensión 3 del cuestionario. 


\section{5.-Comparaciones entre establecimientos $\mathrm{A}$ y $\mathrm{B}$ y las dimensiones.}

Mediante el cálculo de estadísticos muestrales de tendencia central y dispersión, es posible observar que los encuestados de la escuela A tienen una mejor percepción del liderazgo del director que los del establecimiento B, puesto que, en promedio, los puntajes obtenidos en la escala son más altos (ver tabla 10). No se visualizan grandes diferencias en la dispersión de los índices, puesto que la desviación estándar es bastante similar.

Tabla 10

Media y desviación estándar de los puntajes de la escala global del cuestionario para alumnos y apoderados en ambos establecimientos

\begin{tabular}{lll}
\hline & Escuela A & Escuela B \\
\hline Media & 0,89 & 0,83 \\
Desviación estándar & 0,14 & 0,13 \\
\hline
\end{tabular}

Fuente propia

Con respecto a las dimensiones, de la escala, en ambas escuelas la dimensión 2: Consideración individualizada, fue la peor evaluada. En la escuela A, tanto la dimensión 1 que corresponde al Liderazgo carismático, como la 3 que evalúa el Liderazgo inspiracional y la 4 Estimulación intelectual, obtuvieron similar promedio de respuestas afirmativas, mientras que en la escuela B la dimensión 3 que mide el Liderazgo inspiracional y 4 se posicionan como las mejores evaluadas, dejando a la dimensión 1 con un promedio mucho más bajo (ver tabla 11).

Tabla 11

Media de los puntajes de cada dimensión del cuestionario para alumnos y apoderados en ambos establecimientos

\begin{tabular}{lll}
\hline & Escuela A & Escuela B \\
\hline Dimensión 1 & 0,90 & 0,82 \\
Dimensión 2 & 0,78 & 0,72 \\
Dimensión 3 & 0,90 & 0,89 \\
Dimensión 4 & 0,91 & 0,90 \\
\hline
\end{tabular}

Fuente propia

Si se analizan los subgrupos de la muestra, se pude visualizar que en la escuela A los alumnos obtuvieron, en promedio, puntajes más altos que los apoderados; por el contrario, en el establecimiento $B$ son los apoderados quienes obtuvieron puntajes más altos que los estudiantes. En ambos establecimientos, la opinión de los alumnos es más homogénea que la de los apoderados.

Otro factor interesante de destacar sobre el análisis anterior es la similitud en las medias de los apoderados de ambos establecimientos. Esto quiere decir que los apoderados de las 
dos escuelas valoran de la misma manera el liderazgo del director del establecimiento. Por otra parte, los estudiantes sí presentan una diferencia importante en los promedios de los puntajes obtenidos, lo que permite concluir que la escuela $A$ posee un promedio más alto que el $\mathrm{B}$ debido a la opinión de los alumnos de ese establecimiento.

En la tabla 12 puede profundizarse la información sobre los promedios de cada subgrupo en cada dimensión. Es posible advertir que los apoderados de la escuela A puntuaron ligeramente más alto que los de la escuela $B$ en la dimensión 1 Liderazgo carismático, mientras que los apoderados del establecimiento B puntuaron más alto en la dimensión 3 Liderazgo inspiracional y 4 Estimulación intelectual. Se puede constatar nuevamente la gran diferencia entre los promedios de los alumnos de ambos centros, siendo la brecha más grande la presente en la dimensión 1 (0,93 v/s 0,79).

Tabla 12

Media de los puntajes de cada dimensión de la escala en alumnos y apoderados de ambos establecimientos

\begin{tabular}{lllll}
\hline & Escuela A & & Escuela B \\
& Alumnos & Apoderados & Alumnos & Apoderados \\
\hline Dimensión 1 & 0,93 & 0,87 & 0,79 & 0,84 \\
Dimensión 2 & 0,79 & 0,77 & 0,66 & 0,77 \\
Dimensión 3 & 0,94 & 0,86 & 0,85 & 0,92 \\
Dimensión 4 & 0,92 & 0,89 & 0,84 & 0,95 \\
\hline
\end{tabular}

Fuente propia

4.6.-Tercer informe de análisis de resultados en investigación de tesis doctoral. Análisis inferencial de los resultados de las respuestas a las escalas de medición aplicadas.

El presente informe entrega los resultados de los procedimientos de inferencia estadística que fueron realizados sobre los puntajes de cada encuestado. Dichos procedimientos buscan establecer si existen diferencias significativas en el liderazgo educacional ejercido por el director de ambos establecimientos, y si estas diferencias se encuentran a nivel global 0 en algunas de las dimensiones que midieron los instrumentos. El objetivo final es encontrar indicios que permitan sugerir que es la diferencia en el liderazgo de los directores que es uno de los factores que influyen en la brecha de aprendizaje de los estudiantes de ambos establecimientos.

Por ello, la primera parte del informe se dedicará a establecer algunos indicadores que caracterizan ambos establecimientos, permitiendo verificar que ambas escuelas son similares en su composición y estructura, de tal manera que se pudiese insinuar que la brecha en los puntajes SIMCE de ambos establecimientos pueda deberse a la diferencia en el liderazgo ejercido por los directores.

La segunda parte del informe constata si efectivamente existen diferencias significativas en el liderazgo de la dirección de ambas escuelas. En el segundo informe ya se hizo evidente que existen disparidades en los resultados de ambos establecimientos, pero es necesario 
indagar si estos contrastes se deben simplemente al error muestral o si ciertamente existen disimilitudes a nivel poblacional.

Para conseguir este propósito, se efectuaron procedimientos para determinar si se cumplen los supuestos necesarios para realizar inferencia paramétrica. En aquellos casos en que pudo advertirse que los requisitos estaban garantizados, se aplicaron pruebas t de Student. Cuando los supuestos no pudieron comprobarse, se efectuaron procedimientos no paramétricos, en específico, la prueba $U$ de Mann - Whitney. Para cada prueba, se fijó un nivel de significancia de 0,05 . Los valores $p$ asociados a cada contraste fueron aproximados por redondeo a la tercera cifra decimal.

Los procedimientos de inferencia fueron realizados en el programa IBM SPSS Statistics versión 21. El presente informe fue redactado en el programa Word de Microsoft Office 365 ProPlus, versión 1902.

\section{7.-Comprobación de supuestos.}

Se efectuaron pruebas de normalidad de Shapiro - Wilkpara determinar si la distribución de los índices de las escalas globales y de cada dimensión cumple con los supuestos de normalidad (Shapiro, \& Wilk, 1965) para realizar procedimientos paramétricos de comparación de medias, como la prueba t de Student (Quader, 2016).

La prueba de Shapiro - Wilk para el índice total de la escala para directivos y docentes, resultó ser significativa tanto para los encuestados del establecimiento $A(p=0,000)$, como para los del establecimiento $B(p=0,000)$. Es decir, se descarta la hipótesis que los índices posean una distribución normal a un nivel de significancia de 0,05 . Al momento de aplicar la misma prueba para cada una de las dimensiones de la escala, el resultado fue el mismo para los 6 casos: valores $p$ iguales a 0,000 tanto para los índices de los encuestados de la escuela A como para los de la escuela B.

La tabla 13 muestra los valores p para la prueba de Shapiro - Wilk en cada subgrupo del estudio, tanto para la escala global, como para sus dimensiones.

Tabla 13

Valores $p$ en la prueba de Shapiro - Wilk para cada subgrupo de estudio en la escala para directivos y docentes

\begin{tabular}{lllll}
\hline Dimensión & $\begin{array}{l}\text { Escuela A } \\
\text { Directivos }\end{array}$ & Docentes & $\begin{array}{l}\text { Escuela B } \\
\text { Directivos }\end{array}$ & Docentes \\
\hline Dimensión 1 & 0,000 & 0,000 & 0,000 & 0,000 \\
Dimensión 2 & 0,000 & 0,000 & 0,000 & 0,000 \\
Dimensión 3 & 0,000 & 0,000 & 0,095 & 0,006 \\
Dimensión 4 & 0,000 & 0,001 & 0,002 & 0,002 \\
Dimensión 5 & 0,000 & 0,000 & 0,006 & 0,029 \\
Dimensión 6 & 0,005 & 0,000 & 0,008 & 0,010 \\
Escala global & 0,005 & 0,000 & 0,104 & 0,010 \\
\hline
\end{tabular}

Fuente propia 
En el caso de la escala para estudiantes y apoderados, ya que el tamaño de la muestra es suficiente para asegurar los supuestos necesarios para realizar una prueba $t$, no se aplicaron pruebas de normalidad $y$ se decidió efectuar pruebas $t$ en todas las comparaciones.

\section{8.-Comparación entre establecimientos A y B.}

Se compararon las distribuciones de los índices globales de cada encuestado en la escala para directivos y docentes entre ambos establecimientos. El valor $p$ asociado al contraste fue igual a 0,000, lo que indica que existen diferencias significativas en las distribuciones de los puntajes de cada establecimiento. Como pudo observarse en los resultados presentados en el segundo informe, son los encuestados de la escuela A quienes tienden a responder de manera positiva a las aseveraciones de la escala. Por consiguiente, los directivos y docentes de la escuela $A$ afirman que la Dirección de su establecimiento posee mayor liderazgo educativo de lo que afirman los encuestados del establecimiento $B$.

Al comparar los puntajes entre los establecimientos en cada subgrupo del estudio, no se encuentran diferencias estadísticamente significativas en el cuerpo directivo ( $p=0,133)$, pero sí en los profesores de cada establecimiento $(p=0,000)$. La opinión que tiene los directivos de cada escuela es similar en cuanto a al liderazgo del director, pero los profesores de la escuela A tienen una mejor visión del liderazgo del director de su escuela.

Cuando se analizan los subconstructos de la escala, se encontraron diferencias entre los establecimientos, significativas desde un punto de vista estadístico, en todas las dimensiones, excepto en la segunda: La Dirección es capaz de transmitir los fines y prioridades educativas a toda la comunidad escolar. La tabla 31 muestra los valores $p$ de las pruebas realizadas por dimensión, considerando todos los encuestados y cada subgrupo.

Tabla 14

Valores p para la prueba de comparación entre establecimientos de cada dimensión de la escala para directivos y docentes

\begin{tabular}{llll}
\hline Dimensión & Directivos & Docentes & Todos \\
\hline Dimensión 1 & 1,000 & 0,005 & 0,007 \\
Dimensión 2 & 0,300 & 0,611 & 0,408 \\
Dimensión 3 & 0,016 & 0,004 & 0,000 \\
Dimensión 4 & 0,151 & 0,074 & 0,046 \\
Dimensión 5 & 0,193 & 0,001 & 0,001 \\
Dimensión 6 & 0,898 & 0,000 & 0,001 \\
\hline
\end{tabular}

Fuente propia

Dentro de los índices de los directivos, solo se advierte una diferencia significativa en la dimensión 3 que mide "La Dirección apoya a los profesores en el ámbito curricular", mientras que, en los puntajes de profesores, se evidencian diferencias significativas en todas las dimensiones, excepto en la 2: La Dirección es capaz de transmitir los fines y prioridades educativas a toda la comunidad escolar y la 4: La Dirección crea un ambiente ordenado y un clima escolar que facilita la enseñanza y el aprendizaje. 
En consecuencia, el cuerpo directivo del establecimiento $A$ posee una mejor visión de su apoyo a los profesores en el ámbito curricular que los directivos de la escuela $B$ y los profesores de ambos establecimientos tienen similar opinión en lo que respecta a la capacidad de la Dirección de transmitir los fines y prioridades educativas a la comunidad escolar y de crear un ambiente ordenado y un clima escolar que favorece el aprendizaje.

Ahora bien, sobre la escala para apoderados y alumnos, se encontraron diferencias significativas en las medias de los índices de los encuestados de la escuela $A$ con respecto a los encuestados de la escuela $B(p=0,003)$. La muestra del establecimiento A plantea que el liderazgo del director de aquel centro es mejor de lo que piensan sobre su director quienes componen la muestra en la escuela $B$.

Esta diferencia está motivada por la opinión de los alumnos, ya que los datos apuntan a que existe evidencia suficiente para afirmar que los alumnos de la escuela $A$ tuvieron, en promedio, un índice mayor que los del establecimiento $B(p=0,000)$. Por el contrario, la visión de los apoderados sobre el liderazgo del director no presenta disimilitudes significativas $(p=0,614)$.

La tabla 15 muestra los valores p para la comparación de medias en cada dimensión, tanto para la totalidad de los encuestados como para cada subgrupo de estudio.

\section{Tabla 15}

Valores p para la prueba de comparación entre establecimientos de cada dimensión de la escala para apoderados y alumnos

\begin{tabular}{llll}
\hline Dimensión & Apoderados & Alumnos & Todos \\
\hline Dimensión 1 & 0,438 & 0,000 & 0,000 \\
Dimensión 2 & 1,000 & 0,000 & 0,028 \\
Dimensión 3 & 0,082 & 0,005 & 0,526 \\
Dimensión 4 & 0,030 & 0,003 & 0,518 \\
\hline
\end{tabular}

Fuente propia

Puede observarse que la única dimensión donde existen diferencias significativas en las medias de los puntajes de los apoderados entre ambos establecimientos es en la que guarda relación con la estimulación intelectual, donde los apoderados de la escuela $B$ evalúan mejor a su director que los apoderados del establecimiento $\mathrm{A}$. Con respecto a los alumnos, existen diferencias significativas en todas las dimensiones, pero en este caso, son los estudiantes de la escuela A quienes tienen puntajes más altos. En total, existen diferencias significativas en la dimensión 1: Liderazgo carismático y 2: Consideración individualizada a favor del director de la escuela $A$, es decir, los encuestados en esta escala que pertenecen al establecimiento $B$ tienen una visión más crítica de la labor del director en cuanto al liderazgo carismático y la consideración individualizada.

\section{5.-Conclusiones, limitaciones e implicaciones.}

Esta investigación nació como una motivación personal, al observar que alumnos provenientes de escuelas vulnerables de la comuna de Antofagasta, diferían entre sus puntajes SIMCE. 
En esta investigación se planteó el objetivo de determinar qué competencias debe tener un director para promover una escuela efectiva en sectores vulnerables de la comuna de Antofagasta en dos escuelas municipalizadas, concluyendo que se necesita como principal pilar la influencia del director sobre los profesores a través de determinadas prácticas de liderazgo en el establecimiento que permitirán incidir positivamente en los aprendizajes de los estudiantes y el logro de los objetivos trazados. Considerando que estas prácticas se encuentran en un estilo de liderazgo transformacional y que se caracteriza por las siguientes competencias: El director tiene una idea de las necesidades de la escuela siempre centrado en las necesidades de los alumnos, es capaz de transmitir los fines y prioridades educativas a toda la comunidad escolar, construye relaciones con la comunidad, organiza; apoya a los profesores en el ámbito curricular; crea un ambiente ordenado y un clima escolar que facilita la enseñanza y el aprendizaje; conoce la enseñanza de calidad y trabaja activamente con el profesorado para mejorar su capacidad profesional; supervisa y evalúa el rendimiento de la escuela.

Finalizados los análisis descriptivos, puede obtenerse un panorama general de la visión que poseen directivos, docentes, estudiantes y apoderados de ambos establecimientos educacionales. Después de haber revisado los resultados para cada objetivo específico, parece interesante realizar un análisis en el que se pueda revisar los aportes de manera conjunta. De esta forma, se abordará cuánto influye el liderazgo y gestión de los directores que trabajan en contexto de vulnerabilidad y los resultados de aprendizaje de sus estudiantes. Una Escuela Efectiva se entiende como aquella que promueve de forma duradera el desarrollo integral de todos y cada uno de sus alumnos, a través de determinadas prácticas de liderazgo del director, logrando altos resultados en el rendimiento escolar, más allá de lo que sería previsible por su rendimiento inicial y su situación social, cultural y económica.

Concluyendo, nuestro primer objetivo específico fue identificar las competencias que posee el director de una escuela efectiva de sectores vulnerable a través de dimensiones de actuación de gestión y liderazgo.

Hoy se requiere que los líderes escolares tengan la capacidad de transformar sus unidades educativas, motivar y entregar el apoyo necesario con autonomía en su gestión y liderazgo, de tal forma que se traduzca en prácticas inherentes en su quehacer diario en forma constante, permanente y continua para que conduzcan al mejoramiento de los aprendizajes, éstos deberían ir acompañados de modelos de liderazgos, de tal forma que le permita manejar la diversidad de funciones que deben cumplir los directores junto a sus equipos.

El segundo objetivo específico es describir qué elementos del liderazgo posee un director de una escuela efectiva de sectores vulnerables.

En los establecimientos en los que los directores son más comprometidos y responsables, se observa un mayor nivel de práctica efectiva lo que significa no dejar de lado características como estar abiertos a escuchar, ser empático, tener una alta motivación, ser perseverantes. Es necesario que existan estos atributos en los directores, por ser características percibidas por los docentes y demás integrantes de la comunidad escolar, el sentido de eficiencia, compromiso y alta responsabilidad, puede influir de manera importante en todo lo que ocurra en la escuela. 
El tercer objetivo específico es estudiar cómo afectan las dimensiones del liderazgo de una escuela efectiva, en escuelas con bajos resultados SIMCE.

Podríamos decir que no basta con intencionar desde la normativa el rol pedagógico de los directores y sus equipos directivos, más bien esto debería ir acompañado de una redefinición de roles al interior de cada unidad educativa en aquellas escuelas en las que existen bajos niveles de rendimientos, requiere de equipos técnicos con mayor experticia, roles bien estructurados y que los directores puedan concentrar sus prácticas en establecer una dirección clara e informada de las necesidades de la escuela y una vez que la escuela adquiera mayor capacidad, los directores y jefes técnicos, deberian socializar y compartir las tareas técnicas pedagógicas con los docentes en talleres de reflexión pedagógica sobre sus prácticas, comprometiéndolos para el mejoramiento de la enseñanza y el aprendizaje.

El cuarto objetivo específico es analizar qué elementos de liderazgo, desde el enfoque de una escuela efectiva, no se encuentran en un director con bajos resultados SIMCE.

Siguiendo a Bandura (1996), el sentido de eficacia individual dice relación con la creencia personal de que se pueden llevar a cabo acciones exitosamente para conseguir los objetivos. El sentido de eficacia individual tiene dos dimensiones: creer que las acciones que uno realiza ayudarán a conseguir el objetivo deseado y creer que uno tiene la capacidad para realizar esas acciones. El autor también sostiene que la percepción que un individuo tiene respecto a su capacidad, para desarrollar una tarea, tiene una fuerte influencia sobre la cantidad de esfuerzo que invierte, el tiempo en que persiste en tratar de realizar la tarea y cuán resistente es frente a un eventual fracaso. El autor también aclara que el sentido de eficacia, no es una característica personal, sino un sentimiento de confianza respecto a los esfuerzos que se emprenden para realizar una tarea en un contexto específico.

Como en el caso de esta investigación en opinión de los padres, apoderados y estudiantes, consideran como elementos importantes que el director escuche con atención a sus estudiantes, que sea cercano a toda la gente que integra la comunidad escolar, que anime a sus profesores, reconozca y felicite en diversas ocasiones.

La relevancia de las teorías sobre las eficacias directivas y docentes para el liderazgo en Chile son claras. Si los líderes escolares no son capaces de emprender el mejoramiento escolar con éxito, las reformas que se intente implementar para el mejoramiento tendrán baja incidencia.

En general, los encuestados del establecimiento A poseen una mejor opinión sobre el liderazgo del director que aquellos que respondieron el cuestionario en la escuela B. Bajo esta misma línea, es necesario destacar que son los mismos directivos quienes respondieron afirmativamente a las aseveraciones en una mayor proporción, es decir, valoran el liderazgo que realiza el director.

En el establecimiento A existe una visión positiva dentro del personal de la escuela sobre las acciones de liderazgo que el director realiza, ya que todos los directivos y docentes encuestados respondieron afirmativamente al menos a la mitad de las aseveraciones de la escala. Un $78 \%$ de ellos obtuvo un puntaje global superior a 0,9 y en todas las dimensiones más del $50 \%$ respondió positivamente a la totalidad de los ítems (excepto en la dimensión 6 
que corresponde si La Dirección supervisa y evalúa el rendimiento de la escuela/liceo con un $49 \%$ ).

En ambos establecimientos $\mathrm{A}$ y $\mathrm{B}$, la dimensión mejor evaluada fue la número 1 , que corresponde a: «La Dirección tiene una idea clara e informada de las necesidades de la escuela/liceo, siempre centrada en las necesidades de los alumnos(as)». Se observan deficiencias en la manera en que el director del establecimiento $A$ transmiten los fines y prioridades educativas a la comunidad escolar y en la forma en que la Dirección de la escuela B supervisa y evalúa el rendimiento del centro educativo.

Los profesores del establecimiento A tienen una visión mejor del liderazgo del director de su escuela que los profesores de la escuela $B$, excepto en las dimensiones que miden la capacidad de transmitir los fines y prioridades educativas a la comunidad escolar y de crear un ambiente ordenado y un clima escolar que favorece el aprendizaje, donde los profesores de ambos establecimientos poseen similar opinión.

Los directivos de ambos establecimientos perciben de manera similar las competencias de liderazgo del director, en todas las dimensiones que fueron evaluadas, excepto en la capacidad de apoyar a los profesores en el ámbito curricular, donde los directivos de la escuela A perciben que desarrollan una mejor labor que sus pares de la escuela B.

Con respecto al cuestionario para alumnos y apoderados, se observa en ambos establecimientos una tendencia a evaluar de manera positiva el liderazgo inspiracional y la estimulación intelectual del director, mientras que la dimensión de consideración individualizada es la peor evaluada.

Los apoderados de ambos establecimientos tienen opiniones muy similares. Solo en la evaluación de la estimulación intelectual por parte del director se notan diferencias, donde los apoderados del establecimiento B tienen una mejor percepción del director de la escuela. La opinión de apoderados es bastante similar en ambos establecimientos, sin embargo, la de los estudiantes es completamente diferente. En la escuela A los estudiantes poseen una valoración del liderazgo del director mejor que la de los apoderados, mientras que en la escuela B los estudiantes puntúan menos que ellos.

En cambio, los alumnos de la escuela $B$ son más críticos con la labor del director que sus pares de la escuela $A$. Esta diferencia de opinión es la que genera finalmente diferencias significativas en los puntajes de ambos establecimientos, sobre todo en las dimensiones de liderazgo carismático y consideración individualizada.

El Director de una escuela efectiva según la opinión de los padres, apoderados y estudiantes debe llevar a la práctica todas las competencias del Liderazgo transformacional.

Las dimensiones del liderazgo de una escuela efectiva, que no se encuentran en un director de un establecimiento con bajos resultados SIMCE según la opinión de su Equipo de Gestión y docentes son fundamentalmente las del Liderazgo Transformacional.

Los elementos del liderazgo desde el enfoque de una escuela efectiva que no se encuentran en un director con bajos resultados SIMCE, según la opinión de padres, apoderados y estudiantes son que escuche con atención a sus estudiantes, que sea cercano a la gente, 
que anime a sus profesores y estudiantes a ser los mejores en su trabajo, que escucha con atención a sus estudiantes y felicite en diferentes ocasiones.

A través de la presente investigación se demostró que la escuela que se propone vencer las adversidades del medio y hacer que sus estudiantes a pesar de sus desventajas sociales accedan a una educación de calidad, pueden lograrlo con directores que presenten un verdadero liderazgo transformacional para poder obtener efectividad en escuelas de sectores vulnerables.

Con todo, es posible sugerir que las diferencias encontradas entre los liderazgos de los directores, de los establecimientos, pudiese ser una causa de las diferencias en los puntajes SIMCE obtenidos en cada establecimiento, desde que no existen mayores diferencias en otras variables importantes como la composición socioeconómica del alumnado de ambas escuelas 0 en algunas tasas características de los centros educativos.

Es posible visualizar que la hipótesis de normalidad es rechazada en todos los contrastes, excepto para los índices de la dimensión 3: La Dirección apoya a los profesores en el ámbito curricular y de la escala global de los directivos del establecimiento $\mathrm{B}$.

Las dimensiones de liderazgo que posee el director de la escuela efectiva, que para este estudio se denominó A, según la opinión de su Equipo de Gestión, Docentes, Estudiantes, Padres y Apoderados son aquellas que caracterizan el liderazgo transformacional, es decir que el Director debe tener una idea clara de las necesidades de la escuela; ser capaz de transmitir prioridades educativas a toda la comunidad escolar; apoyar a los profesores en el ámbito curricular; crear un ambiente ordenado y un clima escolar que facilite la enseñanza y el aprendizaje; conocer la enseñanza de calidad y trabajo activamente con el profesorado para mejorar su capacidad profesional; supervisar y evaluar el rendimiento de la escuela.

Esta situación finalmente define que la comunidad educativa del establecimiento $\mathrm{A}$ evalúe el liderazgo del director escolar con mejores puntajes que los del establecimiento $\mathrm{B}$.

\section{1.-Aportaciones y limitaciones.}

La metodología utilizada en esta investigación, sirva de base para otras investigaciones ya sea para escuelas municipales de Antofagasta o para los establecimientos de otras comunas de la II Región.

Una vez terminada la investigación, las autoridades de la Corporación Municipal de Desarrollo Social de Antofagasta (COMUDESO), como los directores de los establecimientos analizados, puedan focalizar los planes de acción para el fortalecimiento 0 desarrollo de las competencias y prácticas de liderazgo requeridas por los directores, con el propósito de incrementar la eficiencia y eficacia en sus escuelas. Sistematizar un modelo de sistema de trabajo para el perfeccionamiento de los directores que trabajan en escuelas de sectores vulnerables de la comuna de Antofagasta.

Se espera obtener toda la información completa y precisa de todos los datos recolectados y los análisis estadísticos e informáticos correspondientes a la investigación. Mostrar con 
evidencias los resultados obtenidos a través de tablas, gráficos, esquemas, figuras, textos, etc. y tener las descripciones analíticas.

En esta investigación se pretende estudiar las competencias del director para liderar una escuela efectiva en sectores vulnerables. Se evaluó un conjunto de dimensiones de liderazgo y variables que median el desempeño de los directores, según la información disponible para el contexto escolar chileno. Además, distinguimos cuales de esas prácticas y variables son las más importantes y que pueden impactar los resultados de aprendizajes de los estudiantes.

Se estudiaron algunos conceptos de dimensiones desde el punto de vista cuantitativo tales como si la dirección tiene una idea clara e informada de las necesidades de la escuela centrada en las necesidades de los estudiantes, la dirección es capaz de transmitir los fines y prioridades educativas a toda la comunidad escolar, apoyando a los profesores en el ámbito curricular, creando un ambiente que facilite la enseñanza y el aprendizaje, los directores trabajan activamente con el profesorado para mejorar su capacidad profesional y si existe una evaluación permanente respecto al rendimiento de la escuela. En relación a lo anterior, en el nivel de prácticas de liderazgo podemos afirmar que no solo es importante establecer las dimensiones indicadas, sino que la forma como esas prácticas se realizan deben ser de manera participativa para tener una mayor influencia en el desempeño de los profesores e indirectamente de los estudiantes. De esta manera, en la evaluación de los directores se considera la participación de los docentes directivos, docentes, apoderados y estudiantes, esta participación resulta relevante ya que apoya la teoría existente en relación a la importancia de culturas colaborativas en las escuelas. La relevancia de las teorías sobre eficacia directiva y docente para el liderazgo en Chile es clara. Si los líderes escolares y docentes no se sienten capaces de emprender el mejoramiento escolar con éxito, las reformas que se intenten hacer tendrán escasa o ninguna incidencia.

Desde el punto de vista metodológico, creemos que este trabajo tiene algunos puntos a considerar. Se trabajó con escuelas con similares características en cuanto a su estructura organizacional y se tomaron en cuenta los resultados de mediciones a través de la prueba SIMCE en lenguaje y matemáticas y ambas dependencias con igual nivel socioeconómico.

Los instrumentos de aplicación fueron validados por expertos y programas computacionales lo que podría considerarse una mayor fiabilidad del instrumento. Es importante esclarecer que el uso de diferentes tipos de variables tanto mediadoras y de resultados pueden ayudar a distinguir aquellas variables que más inciden en el liderazgo de los directores, así como la formas practicas mediante las cuales el liderazgo puede incidir en el desempeño docentes y de los estudiantes.

En cuanto a las limitaciones de este estudio es importante que se considere lo siguiente: En primer lugar, este es un estudio de variables relacionadas con el liderazgo escolar y sus efectos desde un enfoque cuantitativo, sin embargo, un estudio sobre el liderazgo escolar en su estricto rigor requeriría de una metodología mixta, que incluya aspectos cualitativos, ya que el liderazgo demanda esencialmente movilizar a otros y de crear capacidades para realizar la tarea educativa. En otras palabras, los estudios cuantitativos no son suficientes respecto a cómo los líderes ejecutan ciertas prácticas y valores de manera efectiva al interior de las escuelas. 
También, hay que señalar las limitaciones respecto a las variables dependiente utilizadas para el caso de los estudiantes, solamente tomamos en cuenta los resultados de los estudiantes de cuartos básicos para lenguaje y matemáticas entendiendo que el liderazgo debería incidir en el aprendizaje de todos los estudiantes en diferentes aspectos de su desarrollo en forma integral.

La muestra de directivos, docentes, estudiantes, padres y apoderada fue adecuada para la investigación. Este trabajo estudió las prácticas del liderazgo de los directores de dos escuelas municipales y están ubicadas las escuelas en sectores vulnerables de la comuna de Antofagasta.

El liderazgo se asocia a la figura del director y deja fuera a los demás líderes escolares y no permite trabajar con las concepciones más modernas sobre el liderazgo distribuido. Los actores que participaron de esta evaluación fueron los equipos directivos, docentes, estudiantes, padres y apoderados como perciben el liderazgo del director al interior de su establecimiento.

\section{2.-Aplicaciones prácticas.}

De los resultados de esta investigación es posible deducir algunas ideas que puedan contribuir al mejoramiento de las dimensiones del liderazgo y gestión de los directores en las escuelas ubicadas en los sectores más vulnerables.

Según Ingvarson y Kleinhenz (2006), un conjunto de estándares comprende valores y principios que deben guiar el trabajo, descripciones de lo que deben saber y hacer los directores eficaces, e indicadores que permitan medir el cumplimiento de estas prácticas. Los estándares sirven como marco para la formación profesional, para la evaluación de los directivos y para postular a los cargos, a través de la alta dirección.

Este trabajo de tesis aporta algunos elementos claves respecto de lo que los directores escolares deberían ser, saber y hacer. Se deja en evidencia la importancia de las emociones directivas en su propio desempeño y en su influencia en los demás integrantes de un equipo directivo. En el caso de los directores deberían prestar atención a su sentido de eficacia a nivel personal y especialmente colectivo, esto significa confiar en los equipos con que cuenta para sacar adelante la escuela y creer que tienen la capacidad de hacerlo. También es importante que aprendan a escuchar a los demás, y con la disponibilidad de abrir espacios de diálogo y participación con el propósito de mejorar los aprendizajes y hacer el cambio al interior de los centros educativos.

Los directores deben tener una mirada pedagógica, de tal forma que puedan ir formando a sus directivos el monitoreo de datos y análisis de las evaluaciones internas como externas de tal forma puedan ir pesquisando en forma oportuna el desempeño de los estudiantes de acuerdo a los niveles correspondientes. En Chile existe la agencia de calidad de la educación, que entrega mayor información de las escuelas respecto a su desempeño en estándares de aprendizajes y sus resultados y tiene la autoridad, para acompañar e intervenir aquellos colegios que no están cumpliendo con los estándares, además exige a los directores dar cuenta pública de su gestión una vez al año. 
Es importante que los directores puedan estar en forma constante motivando a sus equipos directivos para que potencien en los docente prácticas que inciden en los resultados y crear los ambientes propicios para el aprendizaje, desarrollar el sentido de eficiencia, eficacia y efectividad en el trabajo docente y que puedan desarrollar su trabajo de manera comprometida y sientan el apoyo permanente de sus directivos, especialmente cuando se detecten con el monitoreo alumnos que van retrasados en su aprendizaje o presentan algunas necesidades educativas especiales.

Según esta investigación es importante el compromiso y unidad de los docentes para el mejoramiento, así como sentirse apoyado por sus directores y directivos, para potenciar las prácticas para ser capaz de transmitir los fines y prioridades educativas a toda la comunidad, sentirse apoyados en el ámbito curricular, con ambientes disciplinados, propiciando la enseñanza de calidad y mantener el entusiasmo permanente por mejorar su capacidad profesional y ser altamente responsable con los resultados de aprendizajes de los estudiantes dando cuenta pública de su gestión, independientemente de la condición socioeconómica de los estudiantes.

Estos resultados apoyan la idea de formar directores que cuenten con las competencias y que conozcan las diferentes teorías del liderazgo transformacional, de tal forma que cambien su visión administrativa del liderazgo por una visión donde se incorpore el rol pedagógico o transformacional, de manera de dar oportunidades de cambio en las escuelas ubicados en sectores de alta vulnerabilidad y su foco principal sea mejorar sus resultados de aprendizaje en sus estudiantes, impulsar la participación docente y generar espacios de reflexión frente a sus prácticas y la unidad de los docentes en función del mejoramiento permanente, constante y continuo. No se deberían dejar de lado aquellos elementos que forman parte de atributos personales en los directores y que ayudaran en la realización efectiva de las prácticas: capacidad de escuchar, trabajo en equipo, motivación, inspiración, atención individualizada, estimulación intelectual, confianza, comunicación lo que ayudara a la realización efectiva de sus prácticas.

Es importante apoyar a los directores en las tareas administrativas de tal forma que tengan el tiempo suficiente para cumplir con sus roles transformacionales y pedagógicos al interior de las unidades educativas junto a sus equipos directivos y docentes.

\section{6.-Referencias.}

Carbone, R., Olguín, J. C., Ostoic, D., Ugalde, P., y Sepúlveda, L. (2008). Situación del Liderazgo Educativo en Chile. Santiago: MINEDUC - UAH.

Elmore, R. (2010). Mejorando la escuela desde la sala de clases. Santiago de Chile: Área de Educación Fundación Chile.

Fernández, F., Guazzini, C., y Rivera, M. (2012). Caracterización de los directores escolares en Chile. En J. Weinstein y G. Muñoz (Eds.) ¿Qué sabemos sobre los directores de escuela en Chile? Santiago: Fundación Chile.

Gómez, I. (2014). El equipo directivo como promotor de buenas prácticas para la justicia social: hacia un liderazgo inclusivo. Revista Internacional De Educación Para La Justicia Social, 3(2). Recuperado a partir de https://revistas.uam.es/riejs/article/view/343 
Hopkins, D. (2008). Hacia una buena escuela. Experiencias y lecciones. Santiago: Fundación Chile y Fundación CAP.

Leithwood, K. (2009). ¿Cómo liderar nuestras escuelas, Aportes desde la investigación? Área de Educación. Fundación Chile. Santiago: Salesianos.

Marfán, J., Muñoz, G. y Weinstein, J. (2012). Liderazgo directivo y prácticas docentes: evidencia a partir del caso chileno. Chile: Fundación Chile. Recuperado de: http://educacionfch.cl/secciones/directorio-de-recursos/recursos estudios.html?pid=125\&sid=191: Liderazgo-directivo-y-practicas-docentes Evidenciaa-partir-del-caso-chileno

Raczynski, D., Muñoz, G. (2007). Factores que desafían los buenos resultados educativos de escuelas en sectores de pobreza. In: Cueto, Santiago (Ed.).(2007). Educación y brechas de equidad en América Latina. Santiago, Chile: PREAL, 2007. Tomo II, p. 275-352.

Volante, P. (2010). Influencia instruccional de la dirección escolar en los logros académicos. Tesis de Doctorado en Psicología. Pontificia Universidad Católica de Chile.

Weinstein, J., Muñoz, G., Uribe, M., Concha, C., Garay, S., Horn, A., et al. (2011). Liderazgo Directivo y Calidad de la Educación en Chile. Santiago: Fundación Chile. 